\title{
Asymmetric double-well potential for single-atom interferometry
}

\author{
A. I. Sidorov * B. J. Dalton, S. M. Whitlock, and F. Scharnbergi \\ ARC Centre of Excellence for Quantum-Atom Optics and Centre for Atom Optics and Ultrafast Spectroscopy, \\ Swinburne University of Technology, Melbourne, Victoria 3122, Australia
}

(Dated: July 16, 2018)

\begin{abstract}
We consider the evolution of a single-atom wavefunction in a time-dependent double-well interferometer in the presence of a spatially asymmetric potential. We examine a case where a single trapping potential is split into an asymmetric double well and then recombined again. The interferometer involves a measurement of the first excited state population as a sensitive measure of the asymmetric potential. Based on a two-mode approximation a Bloch vector model provides a simple and satisfactory description of the dynamical evolution. We discuss the roles of adiabaticity and asymmetry in the double-well interferometer. The Bloch model allows us to account for the effects of asymmetry on the excited state population throughout the interferometric process and to choose the appropriate splitting, holding and recombination periods in order to maximize the output signal. We also compare the outcomes of the Bloch vector model with the results of numerical simulations of the multi-state time-dependent Schrödinger equation.

PACS numbers: 39.20.+q, 03.75.Dg, 03.75.Be
\end{abstract}

\section{INTRODUCTION}

The evolution of a quantum system in a double-well potential has been the subject of numerous theoretical studies. These include treatments of Josephson-like oscillations [1, 2], dynamic splitting [3] and interference 4] of Bose-Einstein condensates (BECs). The interpretation of these effects is based on the approach [5], in which interference patterns are seen to evolve as a result of successive boson measurements which do not identify the originating condensate. The production of cold atoms and $\mathrm{BEC}$ in microtraps on atom chips 6, 7, 8] and in micro-optical systems 9 has stimulated a great interest towards novel implementations of atom interferometers [10, 11, 12, 13, 14, 15] that are based on the use of double-well potentials. Double-well atom interferometers (DWAI) of both the single-atom and the BEC varieties are well suited to implementation on an atom chip. Here microfabricated structures allow a precise control on a sub-micron scale over the splitting and merging processes. The key processes of splitting [16, 17, 18] and merging 19$]$ of cold atomic clouds and even interference of a BEC after splitting in a double well 20, 21, 22 have been already demonstrated. Although the implementation of a DWAI using a BEC can lead to a $\sqrt{N}$ fold enhancement in precision measurements [23], phase diffusion [24] associated with mean field effects is of concern [20]. Double-well interferometry with a single atom can allow a longer measurement time and in this regard has a potential advantage. An on-chip single-atom interferometer can be integrated with the source of atoms in a ground state - the Bose-Einstein condensate - and be used for sensitive measurements of gravitational fields.

\footnotetext{
*asidorov@swin.edu.au

${ }^{\dagger}$ Also at IQO, University of Hannover
}

DWAI may also be applied to measure collisional phase shift induced by the atom-atom interaction, which is useful for quantum computation processes [25].

Two proposed schemes of a single-atom DWAI involve time-dependent transverse [10] and axial [11] splittings of a trapping potential. An atom is initially prepared in the ground state of a single symmetric trapping potential, which is then split into a symmetric double well. A spatially-asymmetric potential is then applied and nonadiabatic evolution leads to transitions between ground and excited states. The asymmetry is then switched off and the double well is recombined into the original potential. The population of the excited state measures the effect of the asymmetric potential. The DWAI can be considered as a quantum-state Mach-Zehnder interferometer where the evolution of the quantum state via the two separated wells is analogous to the propagation of an optical field via two pathways.

However, these treatments ignore the effect of asymmetry during the splitting and merging stages. In reality asymmetry is always present and could be the result of imperfect horizontal splitting (introducing a gravitybased asymmetry), external spatially-variable magnetic and electric fields or different left and right trap frequencies. We show that the presence of small asymmetries have dramatic consequences on the interferometric process. We have produced a simple model in terms of a Bloch vector evolution that enables us to consider a splitting-holding-merging sequence involving a double well and takes into account the presence of asymmetry at all stages. The two key parameters are the energy gap between the lowest two states of the symmetric component of the trapping potential and an asymmetry parameter, which is related to matrix elements of the asymmetric component of the potential. Non-adiabatic evolution only occurs during the splitting and recombining stages when the torque vector changes much more rapidly compared to the Larmor precession of the Bloch vector. It is 
important that the torque vector remains constant during the holding stage, and that this period is long compared to the splitting and recombination times. In this case the final excited state population is a sinusoidal function of the holding time, with a period determined via the asymmetry parameter.

In this paper we consider the dynamics of a single atom in an asymmetric DWAI, with the basic theoretical framework being outlined in Section II. Using a twomode approximation we develop a Bloch vector model for time-dependent DWAI (Section III) that provides an adequate description of the dynamics of the splitting, holding and recombination processes in the presence of an asymmetric component, and is then used (Section IV) in describing the interferometric process. The validity of the two-mode approach is explored in Section $\mathrm{V}$ through the comparison of predictions of the Bloch vector model with the results of direct numerical simulations of the timedependent multi-mode Schrödinger equation. A discussion of results follows in Section VI and includes a novel scheme to measure the population of the excited state. Theoretical details are dealt with in the Appendix.

\section{THEORETICAL FRAME}

In general, the evolution of a single atom in an interferometer must be described using a three dimensional quantum treatment. However, for a system of cylindrical symmetry (as is present in typical atom chip experiments) it is possible to ignore excitations associated with the two tightly confined dimensions, as long as the dynamics throughout the process is restricted to the dimension of weak confinement (longitudinal splitting). In this system it is possible to reduce the quantum treatment to that of a one dimensional problem.

We consider the one-dimensional evolution of a single atom system due to a time-dependent Hamiltonian $\widehat{H}(t)$ that can be written as the sum of a symmetric Hamiltonian $\widehat{H_{0}}(t)$ and an asymmetric potential $\widehat{V_{a s}}(\widehat{x})$

$$
\begin{aligned}
\widehat{H}(t) & =\widehat{H_{0}}(t)+\widehat{V_{a s}}(\widehat{x}) \\
& =\frac{\widehat{p}^{2}}{2}+\widehat{V_{0}}(\widehat{x}, t)+\widehat{V_{a s}}(\widehat{x}), \\
\widehat{V_{0}}(\widehat{x}, t) & =\left\{1+\left[\beta(t)-\frac{\widehat{x}^{2}}{2}\right]^{2}\right\}^{1 / 2},
\end{aligned}
$$

where a specific form for the symmetric potential $\widehat{V_{0}}$ is chosen [26]. The Hamiltonian and other physical quantities have been written in dimensionless quantum harmonic oscillator units associated with atomic mass $m$ and angular frequency $\omega_{0}$. With the original quantities denoted by primes we have

$$
\widehat{x}=\frac{\widehat{x}^{\prime}}{a_{0}}, \quad \widehat{p}=\frac{a_{0}}{\hbar} \widehat{p}^{\prime},
$$

$$
t=\omega_{0} t^{\prime}, \quad a_{0}=\sqrt{\frac{\hbar}{m \omega_{0}}} .
$$

The dimensionless Hamiltonians, potentials and energies are obtained by dividing the original quantities by $\hbar \omega_{0}$. $\widehat{V_{a s}}$ will be taken as a linear function of $\widehat{x}$.

The symmetric potential depends on a time-dependent splitting parameter $\beta$, whose change from zero to a large value and back to zero again conveniently describes the splitting, holding and recombination processes with periods $T_{s}, T_{h}$ and $T_{r}$ respectively. For zero $\beta$ the symmetric potential involves a single quartic well. When it is large a double harmonic well appears with a separation between minima of $2 \sqrt{2 \beta}$. For zero $\beta$ and for large $x$ the symmetric potential approximates that for a quantum harmonic oscillator with frequency $\omega_{0}$ and mass $m$. Key results in the paper would still apply if other suitable forms for the symmetric potential are used.

We denote the eigenvectors of $\widehat{H}$ as $\left|\phi_{i}\right\rangle$ and their energy eigenvalues as $E_{i}$, where $i=0,1,2, \ldots$ and $E_{i+1}>E_{i}$. The corresponding quantities for the symmetric component of the Hamiltonian, $\widehat{H_{0}}$, will be denoted $\left|S_{i}\right\rangle$ and $E_{S i}$. Both sets of eigenvectors are orthonormal, and all energies and eigenvectors are time dependent. $\widehat{H_{0}}$ is symmetric and the ground state $\left|S_{0}\right\rangle$ is symmetric and denoted as $|S\rangle$, whilst the first excited state $\left|S_{1}\right\rangle$ is antisymmetric and denoted as $|A S\rangle$. Their energies are denoted $E_{S}$ and $E_{A S}$. The one-dimensional nature of the system allows real eigenfunctions $\phi_{i}(x), S_{i}(x)$ to be chosen. In this case the geometric phase 27] is zero.

We can illustrate the general behavior of the lowest few energy eigenvalues $\left(E_{0}, E_{1}, E_{2}, E_{3}, ..\right)$ of the total Hamiltonian $\widehat{H}$ as the splitting parameter is increased from zero to a large value and back. At the beginning and the end of the process when $\beta=0$ the energy eigenvalues are well separated. Here the effect of asymmetry $\widehat{V_{a s}}$ is small and the eigenvalues and eigenvectors resemble those for the symmetric Hamiltonian. When the splitting parameter increases and the trapping potential changes to a double well, pairs of eigenvalues ( $E_{0}$ and $E_{1}, E_{2}$ and $E_{3}$, etc) become very close. At this stage the quantum system is very sensitive to the presence of $\widehat{V_{a s}}$ which breaks the symmetry, allows transitions between $\left|\phi_{0}\right\rangle$ and $\left|\phi_{1}\right\rangle$ to occur and causes the eigenvectors $\left|\phi_{0}\right\rangle$ and $\left|\phi_{1}\right\rangle$ (as well as $\left|\phi_{2}\right\rangle$ and $\left.\left|\phi_{3}\right\rangle\right)$ to be localized in the individual wells in this far split regime.

Initially, the atom is prepared in the lowest energy eigenstate $\left|\phi_{0}\right\rangle$ of the single well. Transitions to excited states are suppressed if the time scale for splitting and recombination is much longer than the inverse frequency gap between the relevant states. The energy gap between $E_{0}$ and $E_{2}$ is always larger than the gap between $E_{0}$ and $E_{1}$, and by choosing appropriate time scales we can isolate the two lowest energy eigenstates $\left(\left|\phi_{0}\right\rangle\right.$ and $\left.\left|\phi_{1}\right\rangle\right)$ from higher excited states $\left(\left|\phi_{2}\right\rangle,\left|\phi_{3}\right\rangle\right.$, etc), but still allow for transitions between the two lowest energy eigenstates to occur. As a consequence the dynamics of the DWAI can be treated under the two-mode approxima- 
tion, in which only the two lowest energy eigenstates of the total Hamiltonian $\widehat{H}$ and the symmetric Hamiltonian $\widehat{H_{0}}$ need to be considered. In this case the first excited state probability (a measurable quantity) can vary from zero to one. A proposal for measuring the excited state population is outlined in Section VI.

Using the two-mode approximation expressions for the lowest two energy eigenvalues $\left(E_{0}, E_{1}\right)$ and eigenvectors $\left(\left|\phi_{0}\right\rangle,\left|\phi_{1}\right\rangle\right)$ for the Hamiltonian $\widehat{H}$ will be obtained. A standard matrix mechanics approach will be used, but instead of using the symmetric potential energy eigenvectors $|S\rangle,|A S\rangle$ as basis vectors, we use the left, right (L-R) basis vectors $|L\rangle,|R\rangle$, which are defined by

$$
\begin{aligned}
& |L\rangle=\frac{1}{\sqrt{2}}(|S\rangle+|A S\rangle), \\
& |R\rangle=\frac{1}{\sqrt{2}}(|S\rangle-|A S\rangle) .
\end{aligned}
$$

The states $|L\rangle,|R\rangle$ are orthonormal and for large $\beta$ correspond to an atom localized in the left or right well, respectively. However, even for a single well the $L-R$ basis is still applicable. The matrix for the Hamiltonian $\widehat{H}$ in the $L-R$ basis is given by

$$
[\widehat{H}]^{L-R}=\frac{1}{2}\left(E_{S}+E_{A S}\right)\left(\begin{array}{ll}
1 & 0 \\
0 & 1
\end{array}\right)+\frac{1}{2}\left(\begin{array}{cc}
-V_{a s} & -\Delta_{0} \\
-\Delta_{0} & +V_{a s}
\end{array}\right),
$$

where the order of the columns and rows is $L, R$ and we define the convenient real quantities

$$
\begin{aligned}
\Delta_{0} & =E_{A S}-E_{S}, \\
V_{a s} & =\left\langle R\left|\widehat{V_{a s}}\right| R\right\rangle-\left\langle L\left|\widehat{V_{a s}}\right| L\right\rangle \\
& =-\left(\left\langle S\left|\widehat{V_{a s}}\right| A S\right\rangle+\left\langle A S\left|\widehat{V_{a s}}\right| S\right\rangle\right) .
\end{aligned}
$$

The derivation of the Hamiltonian matrix uses the symmetry properties of $|S\rangle,|A S\rangle$ and the reality of the eigenfunctions. The total energy for the symmetric Hamiltonian is given by $E_{S}+E_{A S}$, and the energy gap is given by $\Delta_{0}$. The quantity $V_{a s}$ describes the asymmetry of the system, and would be zero if the Hamiltonian was symmetric. The second equation relates $V_{a s}$ to offdiagonal elements of the asymmetric contribution to the Hamiltonian, indicating its role in causing transitions between the eigenstates $|S\rangle,|A S\rangle$ of the symmetric Hamiltonian. The Hamiltonian matrix (5) is analogous to that for a two-level atom interacting with a monochromatic light field 28]. The symmetric Hamiltonian transition frequency $\Delta_{0}$ is analogous to the Rabi frequency, whilst the quantity $V_{a s}$ is analogous to the detuning.

The energy eigenvalues for the total Hamiltonian $\widehat{H}$ are obtained from the determinental equation as the eigenvalues of the matrix $[\widehat{H}]^{L-R}$, and are given by

$$
\begin{aligned}
& E_{0}=\frac{1}{2}\left(E_{S}+E_{A S}\right)-\frac{1}{2} \Delta, \\
& E_{1}=\frac{1}{2}\left(E_{S}+E_{A S}\right)+\frac{1}{2} \Delta,
\end{aligned}
$$

where the quantity $\Delta$ gives the energy gap for the total Hamiltonian $\widehat{H}$ and is defined by

$$
\Delta=\sqrt{\Delta_{0}^{2}+V_{a s}^{2}}=E_{1}-E_{0} .
$$

In terms of the laser-driven two-level atom analogy, $\Delta$ would be analogous to the generalized Rabi frequency.

The orthonormal energy eigenvectors for the total Hamiltonian $\widehat{H}$ are given by

$$
\begin{aligned}
& \left|\phi_{0}\right\rangle=\sqrt{\frac{1+V}{2}}|L\rangle+\sqrt{\frac{1-V}{2}}|R\rangle, \\
& \left|\phi_{1}\right\rangle=\sqrt{\frac{1-V}{2}}|L\rangle-\sqrt{\frac{1+V}{2}}|R\rangle,
\end{aligned}
$$

where the effect of asymmetry is now represented by the variable

$$
V=\frac{V_{a s}}{\Delta} .
$$

On substituting for $|L\rangle,|R\rangle$ the eigenvectors for the total Hamiltonian can be related to those for the symmetric Hamiltonian $\widehat{H_{0}}$. At the beginning and end of the interferometer process we find that the asymmetry parameter $V_{a s}$ is small compared to the symmetric energy gap $\Delta_{0}$. For $V \ll 1$ the eigenvectors $\left|\phi_{0}\right\rangle,\left|\phi_{1}\right\rangle$ become similar to $|S\rangle$ and $|A S\rangle$ respectively. For $V_{a s} \gg \Delta_{0}(V \simeq 1)$, the eigenvectors $\left|\phi_{0}\right\rangle,\left|\phi_{1}\right\rangle$ become equal to $|L\rangle,|R\rangle$ respectively, the localized eigenvectors for the separate wells.

The behavior of the quantities $\Delta, V_{a s}$ and $\Delta_{0}$ as the splitting parameter $\beta$ is changed is shown in Fig. 1 for the case where the asymmetric potential $V_{a s}(x)$ varies linearly with the coordinate $x$, specifically with $\widehat{V_{a s}}=0.02 \widehat{x}$. The symmetric energy gap $\Delta_{0}$ becomes close to zero for $\beta \gtrsim 4$ and then the actual energy gap $\Delta$ is approximately given by $V_{a s}$. The energy eigenfunctions $\phi_{0}(x)$ and $\phi_{1}(x)$ for different splitting parameters $\beta$ are depicted in Fig. [2] again with $\widehat{V_{a s}}=0.02 \widehat{x}$. The behavior of the potential $V(x)=V_{0}(x)+V_{a s}(x)$ is also shown. For small $\beta$ [Fig. 2(a)] the potential is a single well and the eigenfunctions are approximately symmetric and antisymmetric. For larger $\beta$ [Fig. 2(c)] the potential is a double well, which still appears to be symmetric. However, even with a small asymmetry in the potential the eigenfunctions are no longer symmetric and antisymmetric, but instead are each localized in separate wells. This sensitivity of the eigenfunctions to a small asymmetry is critical to the performance of the present interferometer.

\section{BLOCH VECTOR MODEL}

We can express a general time-dependent normalized state vector $|\Psi\rangle$ as a quantum superposition of the states $|L\rangle$ and $|R\rangle$

$$
|\Psi(t)\rangle=C_{L}|L\rangle+C_{R}|R\rangle .
$$




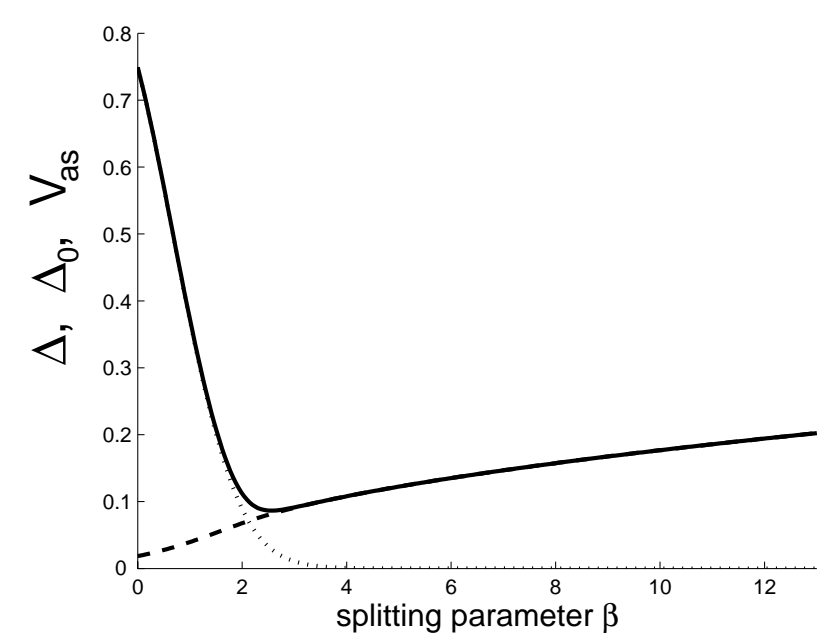

FIG. 1: Energy difference $\Delta$ between ground and first excited states (solid line) for $\widehat{V_{a s}}=0.02 \widehat{x}$ as a function of the splitting parameter $\beta$. Dotted line - energy difference $\Delta_{0}$ for symmetric Hamiltonian, dashed line - asymmetry quantity $V_{a s}$.

Our interferometer will be described in terms of the Bloch vector and its dynamics determined via Bloch equations. We now introduce Pauli spin operators and the Bloch vector. Time-dependent Pauli spin operators $\widehat{\sigma_{a}}(a=x, y, z)$ are defined in the Schrödinger picture

$$
\begin{aligned}
& \widehat{\sigma_{x}}=(|R\rangle\langle L|+| L\rangle\langle R|), \\
& \widehat{\sigma_{y}}=\frac{1}{i}(|R\rangle\langle L|-| L\rangle\langle R|), \\
& \widehat{\sigma_{z}}=(|R\rangle\langle R|-| L\rangle\langle L|) .
\end{aligned}
$$

From its matrix representation in the $L-R$ basis (5), the dimensionless Hamiltonian operator $\widehat{H}$ in the Schrödinger picture can be expressed in terms of the Pauli spin operators as

$$
\widehat{H}=\frac{1}{2}\left(\Omega_{0} \widehat{1}+\Omega_{x} \widehat{\sigma_{x}}+\Omega_{y} \widehat{\sigma_{y}}+\Omega_{z} \widehat{\sigma_{z}}\right),
$$

where

$$
\begin{aligned}
& \Omega_{0}=\left(E_{S}+E_{A S}\right), \\
& \Omega_{x}=-\Delta_{0}, \quad \Omega_{y}=0, \quad \Omega_{z}=V_{a s} .
\end{aligned}
$$

It is convenient to introduce a so-called torque vector, defined as $\vec{\Omega}=\left(\Omega_{x}, \Omega_{y}, \Omega_{z}\right)$.

The Bloch vector is defined to have components which are the expectation values of the Pauli spin operators $\widehat{\sigma_{a}}$ in the quantum state $|\Psi\rangle$. These components will be denoted as $\sigma_{a}$. Hence in the Schrödinger picture

$$
\sigma_{a}=\left\langle\Psi(t)\left|\widehat{\sigma_{a}}(t)\right| \Psi(t)\right\rangle \quad(a=x, y, z) .
$$

The Bloch vector is defined as $\vec{\sigma}=\left(\sigma_{x}, \sigma_{y}, \sigma_{z}\right)$. The Bloch components are bilinear functions of the amplitudes $C_{L}$ and $C_{R}$.
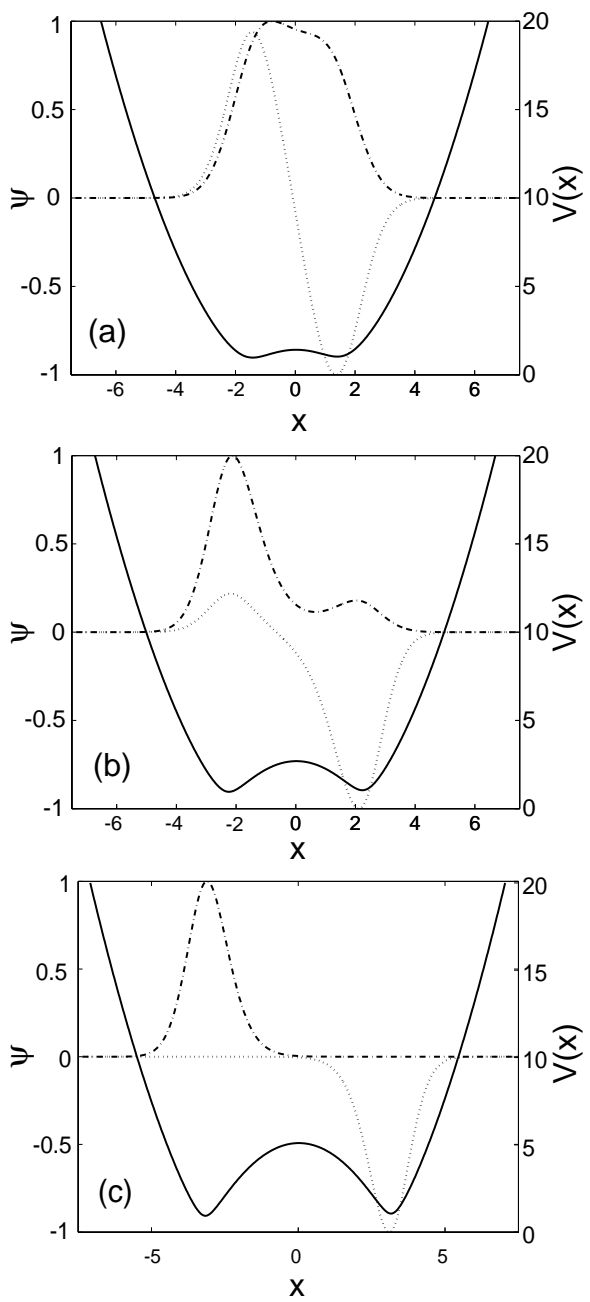

FIG. 2: Stationary eigenfunctions of the ground state (dashed-dotted line) and the first excited state (dotted line) for different values of the splitting parameter $\beta=1$ (a), 2.5 (b) and $5(\mathrm{c})$. The potential $V(x)$ is shown as the solid line.

The evolution of the DWAI system is now described by a set of real variables $\sigma_{x}, \sigma_{y}, \sigma_{z}$ and each of these variables has a certain physical meaning. The component $\sigma_{z}$ is a measure of the imbalance of the atomic population of the localized states $|L\rangle,|R\rangle$. The component $\sigma_{x}$ is a measure of the atomic population imbalance between the delocalized states $|S\rangle,|A S\rangle$, as can be seen if the quantum state is expanded in the symmetric basis. For $\sigma_{x}=+1$ all the population is in the symmetric state $|S\rangle$, for $\sigma_{x}=-1$ it is all in the antisymmetric state $|A S\rangle$. It is thus a measure of the excitation of the first excited state in the unsplit trap regime.

Equations for the components of the Bloch vector can be obtained from Heisenberg equations for the Pauli spin operators. The derivation must take into account the present situation where the Pauli spin operators are explicitly time dependent since the basis vectors $|L\rangle,|R\rangle$ change with time. This differs from the standard situation of time independent basis vectors 28, 29]. How- 

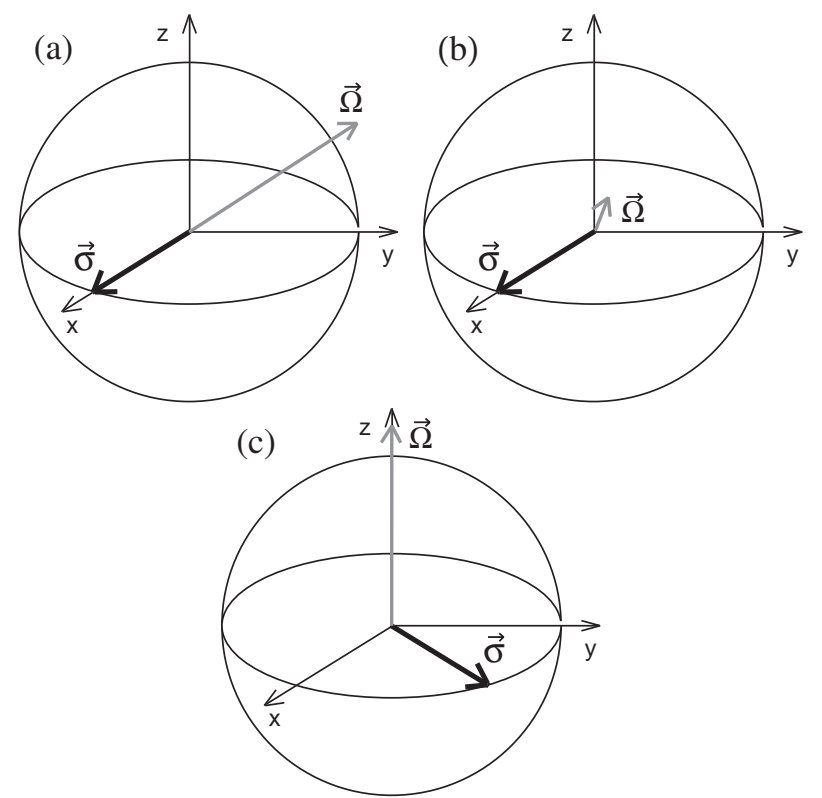

FIG. 3: Evolution of the Bloch vector $\vec{\sigma}$ and the torque vector $\vec{\Omega}$ at different moments of the splitting stage: (a) - at the beginning when $\Delta_{0} \gg V_{a s}$ and $\vec{\Omega} \approx\left(-\Delta_{0}, 0,0\right)$ (b) - when $\Delta_{0}=V_{a s},(\mathrm{c})$ - when $V_{a s} \gg \Delta_{0}$ and $\vec{\Omega} \approx\left(0,0, V_{a s}\right)$.

ever, the additional term in the Heisenberg equations can be shown to contribute zero to the Bloch equations due to the two eigenfunctions in the symmetric basis being real and having opposite symmetry (see Appendix). The Bloch equations are given by $\left(d_{t} \equiv d / d t\right)$

$$
\begin{aligned}
d_{t} \sigma_{x} & =-V_{a s} \sigma_{y}, \\
d_{t} \sigma_{y} & =V_{a s} \sigma_{x}+\Delta_{0} \sigma_{z}, \\
d_{t} \sigma_{z} & =-\Delta_{0} \sigma_{y},
\end{aligned}
$$

and can be solved numerically using the Runge-Kutta algorithm. In vector notation the Bloch equations are

$$
d_{t} \vec{\sigma}=\vec{\Omega} \times \vec{\sigma} .
$$

This form of the Bloch equations is a direct consequence of the equivalence of the two-mode double-well interferometer to a spin $1 / 2$ system. The Bloch vector precesses at the Larmor frequency around the torque vector, which in detail is

$$
\vec{\Omega}=\left(-\Delta_{0}, 0, V_{a s}\right) .
$$

If there is no asymmetry, the $x$ component of the Bloch vector remains unchanged, whilst its component in the $y-z$ plane just rotates about the $x$ axis (Fig. (3).

\section{MODEL OF A SINGLE-ATOM DOUBLE-WELL ATOM INTERFEROMETER}

In the single-atom interferometer under consideration the atom is always located in a trapping potential, which changes from a single well to a double well - which in general is slightly asymmetric - and back again to the original single well. The interferometer is used to measure the effects of this asymmetry, the cause of which may be of measurable interest (e.g., as in a gravity gradiometer). The atom is initially in the ground state $\left|\phi_{0}(0)\right\rangle$ of the original unsplit potential, and as $V_{a s}$ is then small compared to $\Delta_{0},\left|\phi_{0}(0)\right\rangle$ is then approximately the same as $|S(0)\rangle$. The population of the excited state at the end of the recombination process is the measurable interferometer output. The probability $P_{1}$ of finding the atom in the upper energy eigenstate at any time is given by

$$
P_{1}=\left|\left\langle\phi_{1} \mid \Psi\right\rangle\right|^{2},
$$

and this will remain zero unless an asymmetry is present together with suitably short splitting and recombining stages for the interferometer process - so that transitions occur between $\left|\phi_{0}\right\rangle$ and $\left|\phi_{1}\right\rangle$ due to the presence of $\widehat{V_{a s}}$.

We find that

$$
\begin{aligned}
P_{1} & =\frac{1}{2}\left(1+\sigma_{z} V-\sigma_{x} \sqrt{1-V^{2}}\right) \\
& =\frac{1}{2}+\frac{1}{2 \Delta} \vec{\Omega} \cdot \vec{\sigma} .
\end{aligned}
$$

At the beginning and end of the interferometer process $V \ll 1$ and hence the probability $P_{1}$ only depends on the $x$ component of the Bloch vector. The probability $P_{1}(T)$ thus depends on how this component has changed from its initial value of 1 . We can therefore describe the dynamical behavior of the single atom interferometer in terms of the evolution of the Bloch vector during the splitting, holding and recombining stages. At the start of the process the Bloch and torque vectors are antiparallel [Fig. [3(a)] and approximately aligned with the $x$ axis. For small values of the splitting parameter $\beta$ the absolute value of the torque vector is mainly determined by the symmetric energy gap $\Delta_{0}$ (Fig. 11) and its direction remains along the $-x$ direction. The position of the Bloch vector remains mostly along the $+x$ direction [Fig. [3(b)] during early stages of the splitting process. When the splitting parameter is increased further the decreasing energy gap $\Delta_{0}$ becomes comparable with and later much smaller than the asymmetry quantity $V_{a s}$. As a result the torque vector rotates in a $x-z$ plane until it is aligned along the $z$ direction $\left[\vec{\Omega} \approx\left(0,0, V_{a s}\right)\right]$. It is important to make this change non-adiabatically so that the Bloch vector does not follow the torque rotation. If the Bloch vector were to follow the changes of the torque vector adiabatically the atom will always stay in the ground state and no interference would be observed.

During the holding stage the torque vector is constant and the Bloch vector precesses around the torque vector with a constant angular velocity $V_{a s}$, and hence both the $x$ and $y$ components oscillate with a period $2 \pi / V_{a s}$ [Fig. 31(c)]. In an ideal double-well interferometer the splitting and recombination stages are short and the value of the $x$ component does not change much during these stages, so that $\sigma_{x}(T)$ (which defines the final 
excited state population) is basically given by its value at the end of the holding period. The simple behavior during the holding period indicates that the excited state population would have a period $2 \pi / V_{\text {as }}$ considered as a function of holding time. A similar description in terms of the evolution of a Bloch vector also applies to the scheme described in Ref. [1], though the dynamical behavior of the Bloch vector is different.

The behavior of the interferometer may also be described in terms of time-dependent states $|L\rangle,|R\rangle$, which during the holding period represent atoms localized in the left and right wells. The interferometer process involves the transition $|S(0)\rangle \longrightarrow|A S(T)\rangle$, which involves two pathways $|S(0)\rangle \longrightarrow|L(T / 2)\rangle \longrightarrow|A S(T)\rangle$ and $|S(0)\rangle \longrightarrow|R(T / 2)\rangle \longrightarrow|A S(T)\rangle$, involving two possible localized intermediate states associated with the left or right wells. The overall transition amplitude is the sum of amplitudes for the two pathways, and depending on the relative phase between these amplitudes either constructive or destructive interference may occur. For maximum contrast it is desirable that the magnitudes of the two partial amplitudes be equal, so that during the holding period the populations of the left and right well states should be about the same. After optimal splitting the $z$ component of the Bloch vector $\sigma_{z}$ should be kept close to zero during the holding period, however a phase difference between the localized states accumulates. Only at the end of the recombination stage this phase is translated into the population of the excited state.

\section{RESULTS OF NUMERICAL SIMULATIONS}

We studied the evolution of a Bloch vector and the population of the excited state by solving Equations (17) numerically. The splitting parameter $\beta$ is changed linearly from zero up to a maximum $\beta_{\max }$ during the splitting period. It is then held constant at $\beta_{\max }$ during the holding period, and then changed linearly to zero during the recombination period. The dynamical behavior of

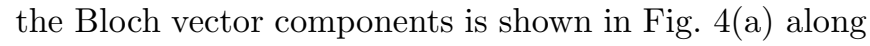
with the time dependence of the asymmetry parameter $V=V_{a s} / \Delta$, the splitting parameter $\beta$ and the excited

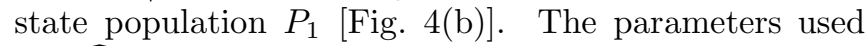
are $\widehat{V_{a s}}=0.02 \widehat{x}, \beta_{\max }=12.5$ and $T_{s}=20, T_{h}=20$, $T_{r}=20$ in dimensionless harmonic oscillator units. Here we observe complex oscillatory behavior for the $x$ and $y$ components of the Bloch vector which occurs during the splitting and merging stages. During the holding stage they exhibit simple periodic variations with frequency $V_{a s}=0.2$. At the same time the $z$ component develops a small negative value during splitting and increases the absolute value even further during merging. The $x$ component reaches a negative value of -0.9 at the end of the process. This corresponds to an excited state population of 0.95 and represents a case of constructive interference.

By monitoring the behavior of $P_{1}$ during the interferometric process we can see when non-adiabatic evolu-
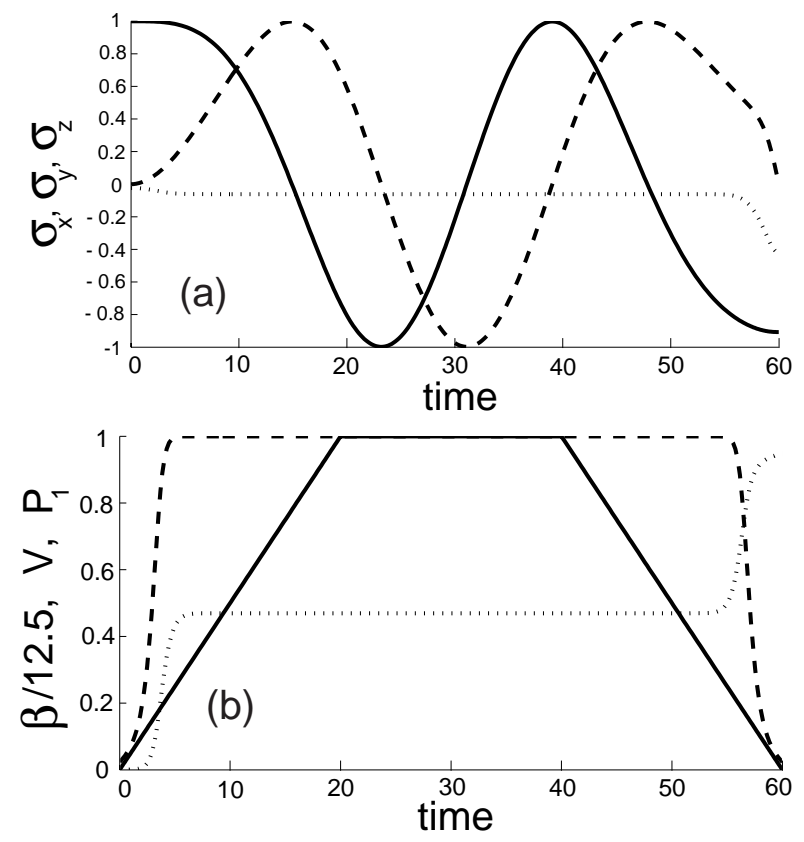

FIG. 4: (a) Time evolution of the Bloch vector components $\sigma_{x}$ (solid line), $\sigma_{y}$ (dashed line) and $\sigma_{z}$ (dotted line) for $T_{s}=T_{h}=T_{r}=20$ and $\beta_{\max }=12.5$; (b) Time evolution of the first excited state population $P_{1}$ (dotted line), the asymmetry parameter $V$ (dashed line) and the splitting parameter $\beta / \beta_{\max }$ (solid line).

tion occurs. The population $P_{1}$ changes from 0 to 0.47 [Fig. [ $4(\mathrm{~b})]$ at the beginning of the splitting process and does not reach the optimal value $1 / 2$ as a result of the non-zero $z$ component of the Bloch vector. The variable $P_{1}$ does not change during the adiabatic precession of the Bloch vector around the torque vector during the rest of the splitting, holding and the beginning of recombining stages. It again exhibits drastic changes in a short period during the re-merging when the torque vector rotates rapidly and the Larmor frequency is relatively small.

It is tempting to limit the evolution of the Bloch vector and the relevant phase accumulation during the splitting and merging stages by making these stages shorter. However this can lead to excitations of higher excited states. We have compared outcomes of the Bloch vector model with the results of the numerical solution of a multi-state Schrödinger equation using the XMDS code [30]. The behavior of the excited state population $P_{1}(T)$ at the end of the interferometer process as a function of the holding period $T_{h}$ is shown in Fig. 5 for the parameters $\widehat{V_{a s}}=0.02 \widehat{x}, \beta_{\max }=12.5, T_{s}=T_{r}=5$ [Fig. 囵(a)], $T_{s}=T_{r}=20$ [Fig. [5(b)] and $T_{s}=T_{r}=200$ [Fig. [5 (c)]. In all cases the sinusoidal behavior of the excited state population as a function of the holding period can be seen. Situations ranging from complete destructive interference to perfect constructive interference are both present. For short splitting times [Fig. [5(a)] we observed a discrepancy between the two models. Multi-state numerical simulations indicate the presence of populated 

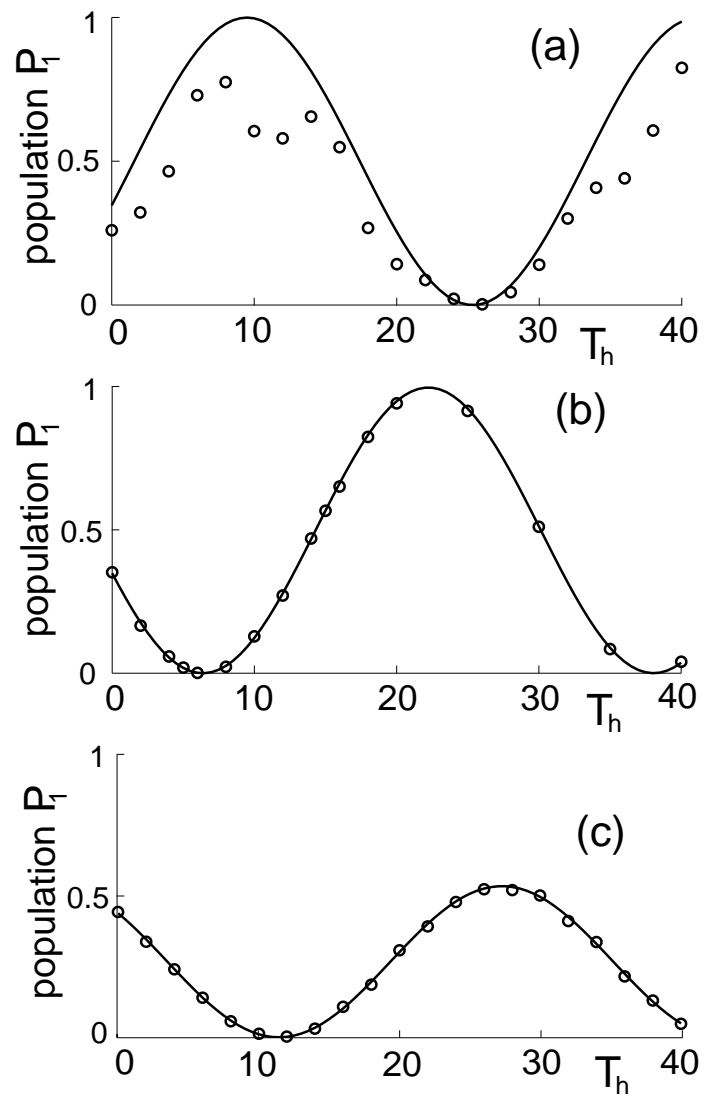

FIG. 5: Dependence of the first excited state population $P_{1}$ at the end of the interferometric process on the duration of the holding stage $T_{h}$ for various durations of the splitting and recombining stages $T_{s}=T_{r}=5$ (a), 20 (b) and 200 (c). Results of the Bloch vector model are represented by solid lines and outcomes of full numerical simulations are presented by circles.

higher energy states which the Bloch vector model ignores. The full numerical calculations show an irregular high frequency modulation of the fundamental frequency signal and a reduced maximum population of the first excited state.

For splitting and merging times $T_{s}=T_{r}=20$ [Fig. [5 (b)] both models show excellent agreement indicating a simple sinusoidal variation of the first excited state population with holding time. This simple behavior is also observed for long splitting time [Fig. [5(c)] but with significantly reduced amplitude of the oscillations. The reduced fringing is attributed to the onset of adiabatic following of the Bloch vector during splitting and recombination which is shown by the presence of a non-zero $\sigma_{z}$ component [Fig. 4(a)]. We noticed that our numerical solution of the Bloch equation is robust with regard to the variations of the signal but is fragile regarding the phase. The error was accumulated during the splitting stage as a result of $V_{a s} \neq 0$ in a merged trap and will scale with the splitting time.

In the asymmetric double-well potential the ground

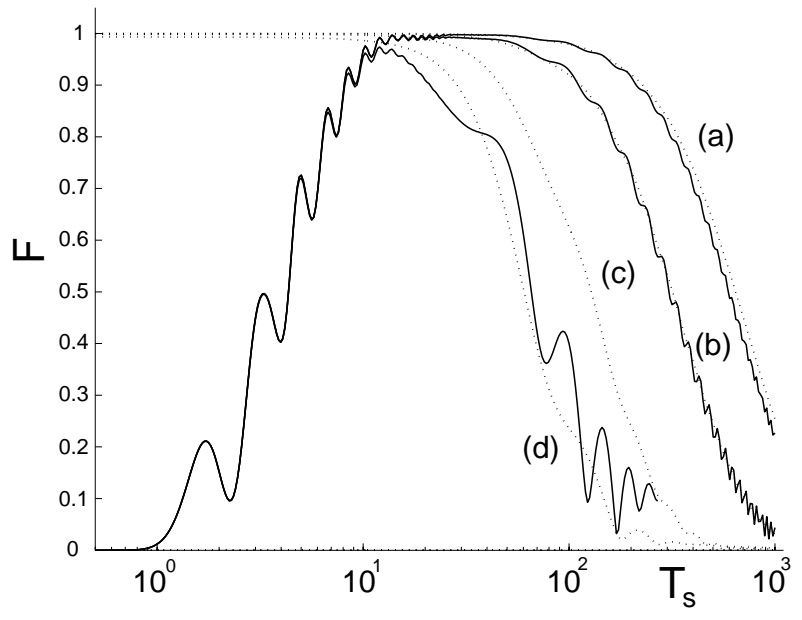

FIG. 6: Dependence of the filling factor $F$ on the duration of the splitting stage $T_{s}$ for $\beta_{\max }=12.5$ and various values of asymmetry $\widehat{V_{a s}}=0.01 \widehat{x}(a), 0.02 \widehat{x}(b), 0.05 \widehat{x}(c)$ and $0.1 \widehat{x}(d)$.

state eigenfunction will predominantly occupy the lower well, and the excited state eigenfunction will be localized in the upper well [Fig. 22(c)]. In the slow splitting regime the onset of the adiabatic evolution will lead to the unbalanced distribution of the atomic wavefunction between the wells, which in turn leads to a reduction in the measured signal. In application to interferometry it can be seen as intrinsic which-way information when the atom will predominantly follow one path after the splitting. In general

$$
|\Psi\rangle=a\left|\phi_{0}\right\rangle+b\left|\phi_{1}\right\rangle+\left|\phi_{i}\right\rangle
$$

where $\left|\phi_{i}\right\rangle$ is a linear combination of all other excited states. We define a filling factor

$$
F=2 a b,
$$

which will describe the balance of ground and excited states populations. The dependence of the filling factor on splitting time is shown in Fig. [6 for a splitting of $\beta=12.5$ and different asymmetries. The results of the Bloch model (dotted line) and the multi-state numerical simulations (solid line) show good agreement for splitting times $T_{s} \geq 20$. For shorter splitting stages the two mode approximation fails and excitations into higher modes take place. In the case of the high asymmetry $\widehat{V_{a s}}=0.1 \widehat{x}[$ Fig. [6(d)] we observe a significant deviation between outcomes of two models. For large values of the asymmetry frequency $V_{a s}$ it is impossible to adiabatically isolate two lower states and the transitions to higher states have to be taken into account.

\section{DISCUSSION AND CONCLUSIONS}

We have applied a Bloch vector model to describe the quantum state interference of a single-atom wavefunction in a time-variable asymmetric double-well potential. 
The probability of finding the atom in the first excited state is closely associated with the magnitude of the spatially dependent external potential and could be used as a measure of the applied asymmetry. Transitions between ground and excited states occur during the splitting and recombination stages. Larmor precession of the Bloch vector during the holding stage is induced by the asymmetry, will effect an interferometric phase and determine the final value of the excited state population. The evolution of the Bloch vector during the splitting and merging stages is also important because it will affect the measurable probability $P_{1}$. We have also shown that special requirements apply to the duration of the splitting and merging stages in order to avoid excitation of higher modes for short times and partial adiabatic following if the splitting is too long. Both these effects lead to a decrease of the measured signal. Interestingly enough they do not affect the contrast of the interference fringes if the first excited state is not initially populated.

Adiabatic evolution of the Bloch vector can offer a new way to measure the first excited state population after the double-well interferometric process. We have already mentioned that in the far-split regime the excited state wavefunction does not overlap with the ground state wavefunction and will predominantly occupy the higher energy well [Fig. 2(c)]. If at the end of the non-adiabatic splitting, phase evolution and non-adiabatic recombination process we also add an additional stage of adiabatic splitting in a known asymmetrical potential, then the wavefunctions of the two states will be spatially separated. For recording the output $P_{1}$ we now simply measure the population of the higher energy well. To shorten the adiabatic evolution time we need to apply the highest available asymmetry (Fig. 6).

\section{Acknowledgments}

We thank T.D. Kieu for fruitful discussions, and T. Vaughan and P. Drummond for the introduction to the XMDS code. This work has been supported by the ARC Centre of Excellence for Quantum-Atom Optics.

\section{APPENDIX: DERIVATION OF BLOCH EQUATIONS}

The state vectors at time $t$ and at time 0 are related via the unitary evolution operator $\widehat{U(t)}$ as $|\Psi(t)\rangle=$
$\widehat{U(t)}|\Psi(0)\rangle$. Operators in the Heisenberg and Schrödinger pictures are related via $\widehat{U}$ as $(\widehat{\Omega})^{H}=(\widehat{U})^{\dagger}(\widehat{\Omega})^{S}(\widehat{U})$. The expectation values of operators in the two pictures are related as $\langle\widehat{\Omega}\rangle=\left\langle\Psi(t)\left|(\widehat{\Omega})^{S}\right| \Psi(t)\right\rangle=\left\langle\Psi(0)\left|(\widehat{\Omega})^{H}\right| \Psi(0)\right\rangle$.

The equation of motion for the Bloch vector components can be derived using the Heisenberg picture via

$$
\frac{d}{d t} \sigma_{a}=\left\langle\Psi(0)\left|\frac{d}{d t}\left(\widehat{\sigma_{a}}\right)^{H}\right| \Psi(0)\right\rangle
$$

where the Heisenberg equation of motion for the Pauli spin operator in dimensionless units is

$$
\frac{d}{d t}\left(\widehat{\sigma_{a}}\right)^{H}=-i\left[\left(\widehat{\sigma_{a}}\right)^{H},(\widehat{H})^{H}\right]+\left(\frac{\partial}{\partial t}\left(\widehat{\sigma_{a}}\right)\right)^{H}
$$

The derivation involves the use of the commutation rules for the Pauli spin operators. For the first term, we have after substituting for $\widehat{H}$ from Eq. (14)

$$
\begin{aligned}
-i\left[\left(\widehat{\sigma_{a}}\right)^{H},(\widehat{H})^{H}\right] & =\frac{-i}{2}\left(\Omega_{0}\left[\widehat{\sigma_{a}}, \widehat{1}\right]+\sum_{b=x, y, z} \Omega_{b}\left[\widehat{\sigma_{a}}, \widehat{\sigma_{b}}\right]\right)^{H} \\
& =\left(\vec{\Omega} \times(\overrightarrow{\hat{\sigma}})^{H}\right)_{a} .
\end{aligned}
$$

Hence the contribution from the first term in Eq. A.2 is given by

$$
\left\langle\Psi(0)\left|\frac{d}{d t}\left(\widehat{\sigma_{a}}\right)^{H}\right| \Psi(0)\right\rangle_{1}=(\vec{\Omega} \times \vec{\sigma})_{a} .
$$

For the contribution from the second term in Eq. (A.2), we may first write $\widehat{\sigma_{a}}=\sum_{A, B=L, R} K_{A B}^{a}|A\rangle\langle B|$, where the $K_{A B}^{a}$ are time independent coefficients that can be read from Eqs. (13), and then

$$
\begin{aligned}
\left(\frac{\partial}{\partial t}\left(\widehat{\sigma_{a}}\right)\right)^{H}= & \sum_{A, B=L, R} K_{A B}^{a} \\
& \times\left[\left(\frac{\partial}{\partial t}|A\rangle\right)\langle B|+| A\rangle\left(\frac{\partial}{\partial t}\langle B|\right)\right]^{H}
\end{aligned}
$$

Using Eq. (12) for the state vector and reverting to the Schrödinger picture we have

$$
\begin{aligned}
\left\langle\Psi(0)\left|\left(\partial_{t}\left(\widehat{\sigma_{a}}\right)\right)^{H}\right| \Psi(0)\right\rangle_{2} & =\left\langle\Psi(t)\left|\left\{\sum_{A, B=L, R} K_{A B}^{a}\left[\left(\partial_{t}|A\rangle\right)\langle B|+| A\rangle\left(\partial_{t}\langle B|\right)\right]\right\}^{S}\right| \Psi(t)\right\rangle \\
& =\sum_{A, B, D=L, R} K_{A B}^{a}\left[C_{D}^{*} C_{B}\langle D|\left(\partial_{t}|A\rangle\right)+C_{A}^{*} C_{D}\left(\partial_{t}\langle B|\right)|D\rangle\right] .
\end{aligned}
$$


To evaluate this result, a consideration of the four quantities $\langle A|\left(\partial_{t}|B\rangle\right)$, where $(A, B=L, R)$ is required, noting also that $\left(\partial_{t}\langle B|\right)|A\rangle=\left(\langle A|\left(\partial_{t}|B\rangle\right)\right)^{*}$. These four quantities can be expressed in terms of related matrix elements in the symmetric basis $\left\langle S_{i}\right|\left(\partial_{t}\left|S_{j}\right\rangle\right)$, where $(i, j=0,1)$. Note $\left|S_{0}\right\rangle \equiv|S\rangle$ and $\left|S_{1}\right\rangle \equiv|A S\rangle$.

Using the normalisation and reality property, we first show that the diagonal terms $\left\langle S_{i}\right|\left(\partial_{t}\left|S_{i}\right\rangle\right)$ are zero. For the off-diagonal terms $\left\langle S_{i}\right|\left(\partial_{t}\left|S_{j}\right\rangle\right)$, these are zero because $\left|S_{i}\right\rangle$ and $\partial_{t}\left|S_{j}\right\rangle$ have opposite symmetry. From these considerations we find that all matrix elements $\langle A|\left(\partial_{t}|B\rangle\right)$, where $(A, B=L, R)$, are zero. Hence the second contribution to the equation of motion for the Bloch vector component is zero

$$
\left\langle\Psi(0)\left|\frac{d}{d t}\left(\widehat{\sigma_{a}}\right)^{H}\right| \Psi(0)\right\rangle_{2}=0
$$

Combining both contributions we find that

$$
\frac{d}{d t} \sigma_{a}=(\vec{\Omega} \times \vec{\sigma})_{a} .
$$

Thus the Bloch equations can be expressed in vector form as in Eq. (18) and in detail as in Eqs. (17).
[1] J. Javanainen, Phys. Rev. Lett. 57, 3164 (1986).

[2] M. W. Jack, M. J. Collett, and D. F. Walls, Phys. Rev. A 54, R4625 (1996).

[3] C. Menotti, J. R. Anglin, J. I. Cirac, and P. Zoller, Phys. Rev. A 63, 023601 (2001).

[4] Y. Castin and J. Dalibard, Phys. Rev. A 55, 4330 (1997).

[5] J. Javanainen and S. M. Yoo, Phys. Rev. Lett. 76, 161 (1996).

[6] R. Folman, P. Krüger, J. Schmiedmayer, J. Denschlag, and C. Henkel, Adv. in Atom., Mol. and Opt. Phys. 48, 263 (2002).

[7] W. Hänsel, P. Hommelhoff, T. W. Hänsch, and J. Reichel, Nature 413, 498 (2001).

[8] H. Ott, J. Fortagh, G. Schlotterbeck, A. Grossmann, and C. Zimmermann, Phys. Rev. Lett. 87, 230401 (2001).

[9] R. Dumke, M. Volk, T. Müther, F. B. J. Buchkremer, G. Birkl, and W. Ertmer, Phys. Rev. Lett. 89, 097903 (2002).

[10] E. A. Hinds, C. J. Vale, and M. G. Boshier, Phys. Rev. Lett. 86, 1462 (2001).

[11] W. Hänsel, J. Reichel, P. Hommelhoff, and T. W. Hänsch, Phys. Rev. A 64, 063607 (2001).

[12] E. Andersson, T. Calarco, R. Folman, M. Andersson, B. Hessmo, and J. Schmiedmayer, Phys. Rev. Lett. 88, 100401 (2002).

[13] J. A. Stickney and A. A. Zozulya, Phys. Rev. A 66, 053601 (2002).

[14] A. Negretti and C. Henkel, J. Phys. B 37, 385 (2004).

[15] H. Kreutzmann, U. V. Poulsen, M. Lewenstein, R. Dumke, W. Ertmer, G. Birkl, and A. Sanpera, Phys. Rev. Lett. 92, 163201 (2004).

[16] D. Cassettari, B. Hessmo, R. Folman, T. Maier, and J. Schmiedmayer, Phys. Rev. Lett. 85, 5483 (2000).

[17] D. Müller, E. A. Cornell, M. Prevedelli, P. D. D.
Schwindt, A. Zozulya, and D. Z. Anderson, Opt. Lett. 25, 1382 (2000).

[18] J. Estève, T. Schumm, J.-B. Trebbia, I. Bouchoule, A. Aspect, and C. I. Westbrook, Eur. Phys. J. D 35, 141 (2005).

[19] W. Hänsel, J. Reichel, P. Hommelhoff, and T. W. Hänsch, Phys. Rev. Lett. 86, 608 (2001).

[20] Y. Shin, M. Saba, T. A. Pasquini, W. Ketterle, D. E. Pritchard, and A. E. Leanhardt, Phys. Rev. Lett. 92, 050405 (2004).

[21] T. Schumm, S. Hofferberth, L. M. Andersson, S. Wildermuth, S. Groth, I. Bar-Joseph, J. Schmiedmayer, and P. Krüger, Nature Physics 1, 57 (2005).

[22] Y. Shin, C. Sanner, G.-B. Jo, T. A. Pasquini, M. Saba, W. Ketterle, D. E. Pritchard, M. Vengalattore, and M. Prentiss, Phys. Rev. A 72, 021604(R) (2005).

[23] M. A. Kasevich, Science 298, 1363 (2002).

[24] J. Javanainen and M. Wilkens, Phys. Rev. Lett. 78, 4675 (1997).

[25] T. Calarco, E. A. Hinds, D. Jaksch, J. Schmiedmayer, J. I. Cirac, and P. Zoller, Phys. Rev. A 61, 022304 (2000).

[26] D. Pulido, Instability in a cold atom interferometer, Master thesis, Worcester Politechnic Institute (2003).

[27] M. V. Berry, Proc. Roy. Soc. London, Ser. A 392, 45 (1984)

[28] L. Allen and J. H. Eberly, Optical Resonance and TwoLevel Atoms (New York: Wiley, 1975).

[29] C. Cohen-Tannoudji, B. Diu, and F. Laloë, Quantum Mechanics, Volume I (New York: Wiley, 1977).

[30] XMDS code is a code generator that integrates equations. It is developed at the University of Queensland, Brisbane, Australia, www.xmds.org. 